\title{
Special issue: Bio-inspired algorithms with structured populations
}

\author{
Bernabé Dorronsoro · Enrique Alba
}

Published online: 3 February 2013

(c) Springer-Verlag Berlin Heidelberg 2013

The advantages of using structured populations in Evolutionary Algorithms (EAs) and other metaheuristicas are well known today, not only for parallelization purposes, but also for enhancing the performance of the algorithm with respect to the equivalent algorithm with panmictic population, even for sequential executions. The use of an appropriate population structure will allow us to create a higher efficiency and efficacy algorithm once structured, both numerically and in real time (e.g. parallelism).

The articles composing this special issue represent excellent novel contributions in this direction. We collected six high-quality papers out of the 43 received ones. These finally selected papers deal with different aspects related to the topic of this special issue, such as theoretical studies, identification of current challenges in the field, novel population structure designs, and empirical validation on highly complex problems.

In our first article, Payne et al. provide a comprehensive overview on the main existing evolutionary algorithms using complex and dynamic population structures. The main results and conclusions are useful, and their limitations are pointed out too. The authors also highlight several open questions related to the use of complex and dynamic population structures, which represent a novel research line.

In the second paper, Lässig and Sudholt propose a theoretical analysis to study the usefulness and correct use of

B. Dorronsoro

University of Lille, Lille, France

e-mail: bernabe.dorronsoro_diaz@inria.fr

E. Alba $(\bowtie)$

University of Malaga, Málaga, Spain

e-mail: eat@1cc.uma.es migration in multipopulation evolutionary algorithms. The way in which information is disseminated across the islands is studied in detail, as well as the effects of the main parameters of such algorithms. The presented theoretical analysis is validated also by an empirical study.

The work of Al-Naqi et al. proposes the use of adaptive three-dimensional population structures for cellular genetic algorithms. The proposed algorithm automatically modifies the population structure to dynamically find the most appropriate exploration-to-exploitation tradeoff at every stage of the search process. This again represents a promising research line for the future of this field.

In the article by Marinakis and Marinaki, authors are presenting a very clever efficient combination of particle swarm optimization (PSO), variable neighborhood search (VNS), and path relinking. The resulting ideas are all included in the form of a new algorithm (PSOENT) that is shown to be very competitive with the state of the art techniques in solving permutation flowshop scheduling problems. The key point is the utilization of an incrementally expanding neighborhood in the swarm, what allows a tradeoff between exploration and exploitation, leading the resulting technique to exhibit a very competitive behavior on standard benchmarks of the target problem.

The paper by Mora et al. is a very interesting one also, combining innovative ideas to solve a complex problem in multi-objetive optimization. Authors go for a bi-objective formulation of TSP and solve many instances by taking advantage of parallel distributed versions of ant colony algorithms. The difficulty of building a competitive distributed ACO is well known in the literature, and authors succeed here to do so in computing quasi-optimum Pareto fronts for the TSP. The analysis of the migration, the realtime enhancements, and the thorough analyses of the 
resulting fronts make this article a real contribution to take into consideration in future works. In addition, the serious statistical validation of the alternative research proposals and the final lessons learned will all cheer the reader to go for this work and enjoy it.

The last paper, by Arnaldo et al., is an excellent manuscript that takes the reader into a journey of methodological studies and real advances of the state of the art, in what is presently known in the domain of distributed algorithms and the evolution of the connecting topology. By crossfertilizating the idea of beta-graphs from complex networks into structured populations, authors perform a thorough analysis of the influence of the algorithm structure into the problem structure itself. The profound study of the idea on benchmarks is conclusive and inspiring for future research, and the achieved conclusions are even further validated on a real problem (a Multi-skill Call Center application).

As guest editors of this issue and researchers, we firmly believe that this special issue importantly contributes to the related literature with relevant and innovative research works. We really hope that the effort we put into the organization of this special issue will be compensated by the interest of the community, and that the selected articles will receive intense citations in the upcoming years because of their interest for researchers in broad fields of theory and practice of complex problem solving. 